\title{
Bézier Modelling of Cracks
}

\author{
Andrew Varley and Peter Rayner \\ Department of Engineering, University of Cambridge, Trumpington Street, \\ Cambridge, CB2 1PZ, UK
}

\begin{abstract}
In this paper we show how arbitrary patterns of cracks can be fitted by Bézier curves of unknown order. The Reversible Jump MCMC technique is used to estimate both the number of curves in the image, and the positions of the knots and control points for each curve. The technique described in this paper is suited to a variety of line fitting applications.
\end{abstract}

\section{Introduction}

There has been a lot of interest recently in statistical image analysis using global image models [1-4], as opposed to pixel-based methods. Here we present an application that is ideally suited to a global parameterisation.

Art historians are keen to be able to establish the period and region of old paintings, as this can help identify fakes. One way of doing this is by examination of the patterns of cracks on the surface of the paint layer [5]. These cracks evolve over many years and are characteristic of the material and paint composition. Research in this area is still in its early stages. Some examples of some actual crack patterns are shown in Fig. 1.
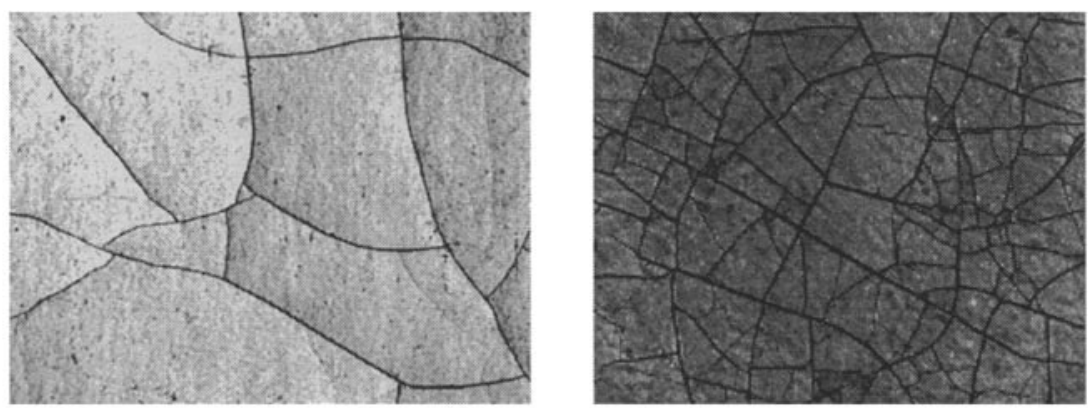

Fig. 1. Examples of crack patterns

\section{The Model}

The crack images $(\mathbf{Y})$ are modelled as an unknown number of Bézier curves, each curve having an unknown number of segments. A Bézier segment is shown in 
Fig. 2 and is completely parameterised by the positions of the 2 endpoints, plus the magnitude, $\mu$, and gradient, $\theta$, of the tangent vectors at the endpoints. A Bézier curve is a number of these segments joined end to end, with the gradients having the same direction (but not necessarily the same magnitude) at the joins (Fig. 3). By varying the number and position of the knots, Bézier curves can be fitted to almost all lines in images.

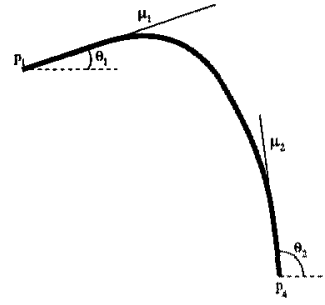

Fig. 2. A Bézier segment

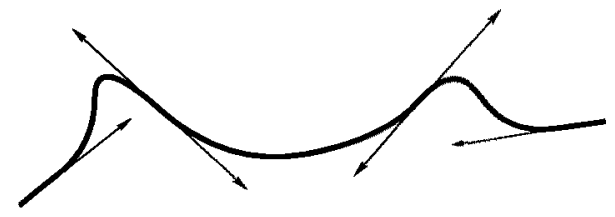

Fig. 3. A Bézier curve

The $i$ th curve is completely parameterised by its width, $w$, greyscale intensity, $c$, the location of the segment endpoints, $P$ (known as "knots" from now on), and the magnitude, $\mu$, and gradient, $\theta$, of the tangent vectors at each knot. These parameters are collected into a single, variable length, parameter vector $\psi_{i}$.

The parameters of the complete model are therefore:

$k$ - the number of curves in the image

$s_{i}$ - the number of knots in the $i$ th curve

$\psi_{i}$ - the curve parameters for the $i$ th curve.

\subsection{The Likelihood}

The likelihood term, $\mathrm{p}(\mathbf{Y} \mid \psi, k)$, is a model of the observational noise present in the original image. We assume that each pixel has independent Gaussian random noise added, the likelihood is therefore

$$
\mathrm{p}(\mathbf{Y} \mid \psi, k)=\frac{1}{\sigma \sqrt{2 \pi}} \exp \left(\frac{1}{2 \sigma^{2}} \sum_{p q}\left(Y_{p q}-S_{p q}\right)^{2}\right)
$$

where the sum is over all the pixels in the image, and $S$ is the model realisation at this iteration. We like to think of the likelihood as a measure of goodness of fit of the model to the image data - if the current model configuration is similar to the original image then the likelihood has a high value, whereas a model configuaration that doesn't match the image very well leads to a low value of the likelihood. 


\subsection{The Priors}

The priors are an indication of the range of the parameters that occur in real images. In this application especially, these are very important in determining the sort of images that get simulated when the algorithm is run. There are two parts to the prior - the first is a prior on the number of curves in the image $(\mathrm{p}(k))$, and the second a prior on the curve parameters, given the number of curves $(p(\psi \mid k))$.

Model Order Prior $(\mathbf{p}(k))$. Prior knowledge of the number of curves in the image is incorporated in the prior $\mathrm{p}(k)$, where we use a Poisson distribution with mean $\lambda_{\mathbf{k}}$,

$$
\mathrm{p}(k)=\frac{\exp \left(-\lambda_{\mathrm{k}}\right) \lambda_{\mathrm{k}}^{k}}{k !}
$$

Curve Prior $\left(\mathbf{p}\left(\psi_{i}\right)\right)$. The number of knots per curve is also modelled by a Poisson distribution,

$$
\mathrm{p}\left(s_{i}\right)=\frac{\exp \left(-\lambda_{\mathrm{s}}\right) \lambda_{\mathrm{s}}^{s_{2}}}{s_{i} !} .
$$

The greyscale intensity of a curve is modelled with a ramp pdf,

$$
\mathrm{p}\left(c_{i}\right)=\operatorname{ramp}(0,255)
$$

This biases towards dark curves, and prevents a large buildup of small bright curves, a similar problem to that noticed by Ripley [6]. The width of a curve is modelled by a Poisson distribution with mean 8 pixels. The knot positions are given a uniform pdf. over the image plane. We have found that reasonable results are obtained by giving the knot positions a non-informative prior like this. We could of course include some sort of repulsion term to prevent there being too many knots too close together, but we haven't really found this to be a problem.

The curve prior for the $i$ th curve, $\mathrm{p}\left(\boldsymbol{\psi}_{i}\right)$, is simply the product of the priors for all the individual curve parameters, and the overall image prior is

$$
\mathrm{p}(\boldsymbol{\psi} \mid k) \mathrm{p}(k)=\mathrm{p}(k) \prod_{i=1}^{k} \mathrm{p}\left(\boldsymbol{\psi}_{i}\right)
$$

\section{The sampler}

Now the model is specified, it remains for it to be fitted to actual images. Since the number of curves present in the image is variable, we have adopted a reversible-jump MCMC sampling scheme to give estimates of both the number of curves present, and their parameters. 
The algorithm begins by "guessing" a random number of curves, in random positions on the image (their parameters are chosen from the respective priors). At each iteration from then on a change is proposed in one or more of the parameters. The change may or may not be accepted, depending on the value of the Metropolis-Hastings-Green acceptance ratio for that particular change,

$$
p(\text { acceptance })=\min (1, \text { likelihood ratio } \times \text { prior ratio } \times \text { proposal ratio } \times|J|)
$$

where the likelihood ratio is the likelihood value for the current model configuartion divided by the likelihood of the previous model configuration, the prior ratio is the current prior value divided by the previous prior value, the proposal ratio is the probability of choosing the reverse step divided by the probability of choosing the forward step, and $|J|$ is the determinant of the Jacobian of the transformation between the old and new parameters.

A detailed derivation of the acceptance probability formula can be found in [7], but all that need concern us here is the fact that if the proposed change leads to a "better" fit between model and image we accept the change. A "worse" fit may or may not be accepted (the fact that we may accept a worse fit means that the algorithm can escape from local minima).

\subsection{The moves in detail}

We have defined fourteen different "moves" that we can propose to make at each iteration. The moves have been designed so that the sampler can easily jump out of local minima. For example, the split type 1 move below is really just a combination of a death of a sequence of knots on one curve, followed by a birth of a new curve. The huge reduction in likelihood resulting from a death of a portion of an almost correct curve means it is highly unlikely to be accepted, hence the curve would never be able to be split.

Curve birth/death (Fig. 4). For a birth, a new curve is added to the model. The width and intensity of the curve are chosen from the respective prior distributions, and the knot and control point positions are chosen randomly (subject to maintaining continuity over the segment joins). A death is the opposite, whereby the curve to be deleted is chosen at random and removed from the model. The proposal ratio for a birth is found to be $\frac{d_{k+1}}{b_{k}(k+1)}$, and for a death $\frac{k b_{k-1}}{d_{k}}$, where $b_{k}\left(d_{k}\right)$ is the probability that a birth (death) move is chosen, given there are $k$ curves currently present in the model. The new parameters introduced in the birth step are accounted for by drawing new random variables from the prior distributions, so the Jacobian is 1 .

Interior knot birth/death (Fig. 5). For a knot birth, a curve is chosen at random, and a position on that curve is chosen at random. A new knot and associated control points are added in that position, so that the shape of the 
curve is unchanged (this is a standard procedure, details of which can be found in [8]). The new knot is then displaced a random distance from its original position and new values for the gradient and magnitude of the tangent vector at the new knot are chosen. A death is the opposite, where we randomly choose an interior knot to be deleted.

Width change. A curve is chosen at random and a new width for that curve is proposed from a uniform distribution centred at the original width.

Intensity change. A curve is chosen at random and a new intensity for that curve is proposed from a uniform distribution centred at the original intensity.

Knot move (Fig. 6). A curve is chosen at random, and a knot on that curve is chosen. A new position for that knot is proposed from a uniform distribution centred on the original position of the knot. The tangent vectors at the moved knot remain the same as before.

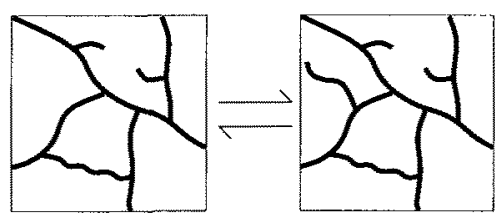

Fig. 4. A curve birth/death

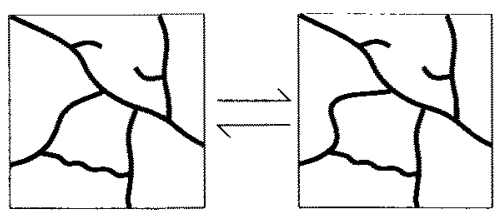

Fig. 6. A knot position change

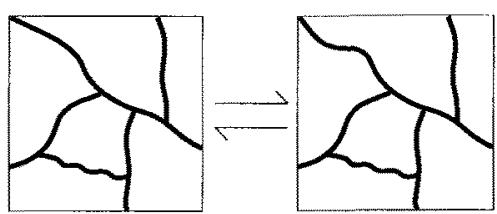

Fig. 5. An interior knot birth/death

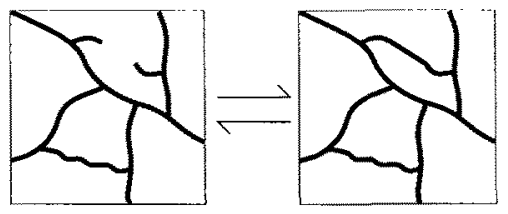

Fig. 7. A split-join type 1 change

Tangent gradient change (Fig. 8). A curve is chosen at random, and a knot on that curve is chosen at random. The tangent gradient at that knot is changed by an angle $\alpha$.

Tangent magnitude change (Fig. 9). A curve is chosen at random, and a knot on that curve is chosen at random. The tangent magnitude of one of the curve segments at that knot is changed. 


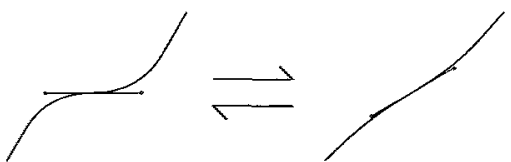

Fig. 8. A tangent gradient change

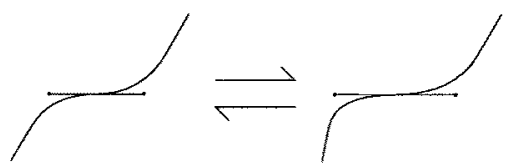

Fig. 9. A tangent magnitude change

Move/gradient combined. This is simply a knot move, followed by a gradient change. We found that much better mixing was achieved by including this combined move.

Split/join type 1 (Fig. 7). Two curves are chosen at random. We propose to join one of the ends of the first curve to one of the ends of the second curve with an additional Bézier segment. The split move is the reverse of this - we propose that a segment of an existing curve gets deleted. The join move introduces 2 new variables into the model (the new segment tangent magnitudes), which are chosen based on the existing tangent magnitudes at the new segment endpoints, plus a random perturbation.

Split/join type 2 (Fig. 10). A curve is chosen at random. If there is another curve endpoint within a circle of radius 50 pixels centred at one of the original curve's endpoints, then we propose to move one of the endpoints to the other. A split move (type 2) is the opposite, so we split a curve in a random place, then propose to move one of the two new endpoints away from the other.
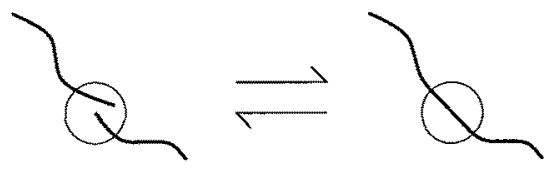

Fig. 10. A split-join type 2 change

Our experience with algorithms of this type has shown that we need to propose births relatively often, as they have a high rejection rate. Deaths should be proposed infrequently, as once a Bézier segment is in the right place, it is not likely to get removed. Various authors have suggested basing the probability of choosing a birth or death on the current value of $k[1,2,7,9]$, however we have found that it makes little difference to have a constant birth and death probability throughout the simulation. We therefore propose a birth with probability 0.2 , a death with probability 0.02 , and all the other moves with equal probability. Due to the huge size of the parameter space, the sampler needs to be run for a large number of iterations, typically a few million. 


\section{Results and Discussion}

We have tested our algorithm on a selection of real data sets, that is digitised close-up photographs of the paint layer of various paintings. Figure 11 shows the original painting, followed by the (random) starting point for the sampler and samples from the posterior at various times afterwards. Examination of the output of the sampler shows that once a curve appears in the model in the correct position on the image, it tends to stay there and grow to adapt to the original curve. The example shown here is typical of the results obtained on other similar images.

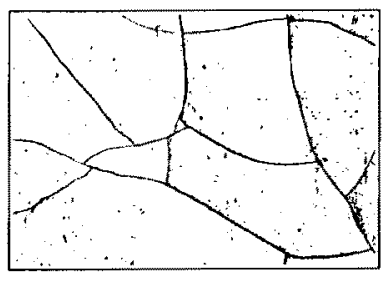

(a) The observed image

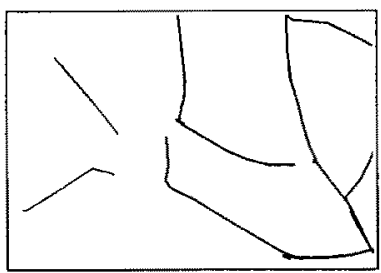

(d) After $5 \times 10^{6}$ iterations

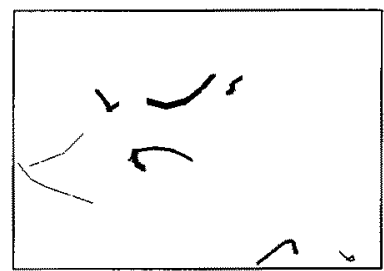

(b) The initial configuration of the sampler

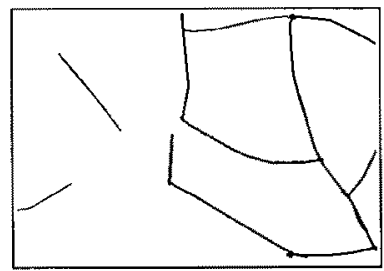

(e) After $10^{7}$ iterations

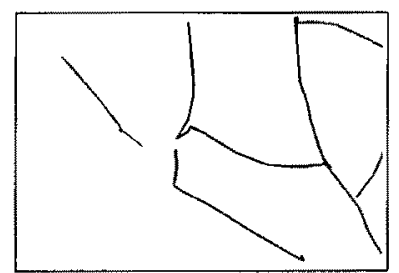

(c) After $10^{6}$ iterations

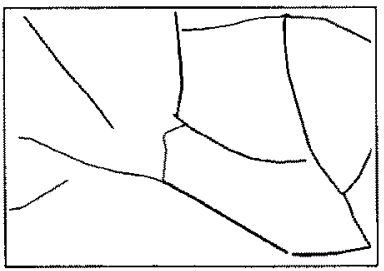

(f) After $8 \times 10^{7}$ iterations

Fig. 11. Samples from the posterior during the simulation

The design of plausible moves is extremely important in algorithms of this kind. In preliminary tests, we had not included the split/join type 2 move. Figure 12 shows the model configuration after $10^{6}$ iterations in this case. It can be seen that there are a number of curves whose ends have not joined where it seems likely that they should have done. The join type 1 move is not very likely in these cases, as it would introduce a large loop which would immediately get rejected, and no other combination of moves is able to overcome the decrease in likelihood arising from deleting one of the existing curves. 


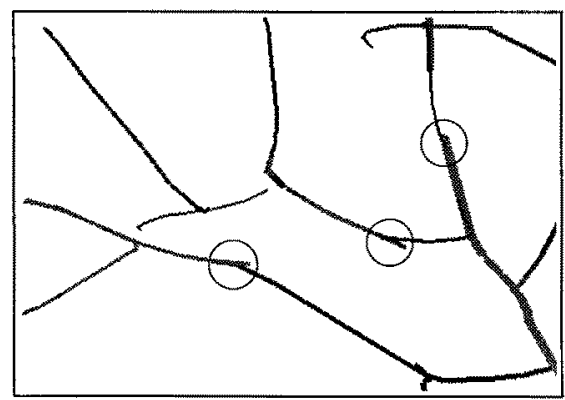

Fig. 12. Sampler output after $10^{6}$ iterations without the split/join type 2 move. The circles show the curve endpoints which wonld be able to join given the existence of the type 2 join move.

Although a couple of million iterations are needed before most of the lines have been detected, it has to be remembered that each iteration requires very little CPU time. The likelihood (which is a pixel by pixel comparison between the model and the observed image) only has to be recalculated at the pixel positions that have actually changed, in this case along the particular line that is being altered. On a SUN Sparc-20, the algorithm runs at approximately 500 iterations per second.

\section{References}

1. Morris, R.: Image sequence restoration using Gibbs distributions. PhD. thesis, Cambridge University, (1995)

2. Rue, H., Syversveen, A. R.: Bayesian object recognition using Baddeley's delta loss. Department of Mathematical Sciences, NTH, Norway, (1995)

3. Clifford, P., Nicholls, G.: A Metropolis sampler for polygonal image reconstruction. Department of Statistics, Oxford University, (1994)

4. Nicholls, G.: Bayesian image analysis with Markov chain Monte Carlo and colored continuum triangulation models. Department of Mathematics, Aukland University, (1996)

5. Bucklow, S.: Formal connoisseurship and the characterisation of craquelure. PhD. thesis, Cambridge University, (1997)

6. Ripley, B., Sutherland, A.: Finding spiral structures in images of galaxies. Philosophical Transactions of the Royal Society of London 332 (1990) 477-485

7. Green, P.: Reversible jump Markov chain Monte Carlo computation and Bayesian model determination. Biometrika 82 (1996) 711-732

8. Foley, J., van Dam, A., Feiner, S., Hughes, J.: Computer graphics: principles and practice. Addison-Wesley (1991)

9. Denison, D., Mallick, B., Smith, A.: Automatic Bayesian curve fitting. Department of Mathematics, Imperial College, (1996) 